\title{
Einige Bildungsfehler der weiblichen Genitalien aus der gynäkologischen Klinik des Prof. Breisky in Prag.
}

\author{
Mitgetheilt
}

von

Dr. Vincenz Johannovsky,

Assistenzarzt daselbst.

1. Defectus vaginae mit Uterus rudimentarius oder gänzlichem Defect des Uterus.

Eva E..., 33jährige ledige Nähterin aus Smichow (klin. Prot. Nr. 512). Gesunde Abstammung. Nie menstruirt; durch wiederholte Krankheiten geschwächt. Leidet bereits seit einer Reihe von Jahren an zeitweiligen Beckenschmerzen.

Befund: Mittelgross, kräftig gebant. Echt weiblicher Typus. Beckenausgang mässig verengt. Oberschenkel stark behart. Aeussere Genitalien normal. Praeputium clitoridis, Frenulum stark markirt. Clitoris selbst nicht vergrössert. Der halbmondförmige Hymen intact. Scheide $1 \mathrm{Cm}$. hinter ihm vollständig abgeschlossen. Per rectum tastet man seitlich und hinten im kleinen Becken mit grösster Deutlichkeit rechts und links je einen länglich geformten, mandelgrossen, beweglichen Körper, welcher vermöge seiner mit charakteristischer, den Follikeln entsprechender, kleinhügeliger Oberfläche und vermöge seiner Druckempfindlichkeit jederseits als functionirendes und vollkommen normal entwickeltes Ovarium gedeutet werden musste. In der Medianlinie zwischen diesen beiden Körpern findet man an Stelle des Uterns ein glattes, erbsengrosses Körperchen, dessen Verbindungen mit Ovarien und atresirter Scheide durch tastbare Bauchfellfalten nicht markirt sind. Einführung eines Katheters in Urethra und Blase und gleichzeitige Rectaluntersuchung ergaben, dass die Zwischenwand zwischen Mastdarm und Katheter mit Ausschluss des kleinen Körperchens in ihrer ganzen Länge dïnn und häutig ist.

Da bei dem vorstehenden Bildungsfehler das kleinwinzige Körperchen eine sichere Deutung in viva nicht gestattete, so blieb in Ermangelung der Autopsie die Frage der rudimentären Entwickelung oder des gänzlichen Defectes unentschieden. Jedenfalls beweist das gleichzeitige Vorkommen normaler Eierstöcke neben ausgesprochener Hemmungsbildung des Gebärorganes, dass uterine Hemmungsbildungen keineswegs von ovariellen begleitet sein müssen. Die zeitwei- 
ligen Beckenschmerzen, über welche die Person klagte, waren, obgleich sie keinen ganz regelmässigen Typus enthielten, sehr wahrscheinlich als Molimina menstrualia zu deuten, da sie dem Sitze nach den Ovarien entsprachen, deren Druckempfindlichkeit zur Zeit der Beschwerden eine gesteigerte war.

\section{Uterus septus bilocularis mit Vagina duplex.}

Anna W...., 17jährige ledige Nähterin aus Smichow (klin. Prot. Nr. 8739). Seit zwei Jahren normal menstruirt. Gesunde Abstammung. Klagt über weissen Fluśs und zeitweilige rechtsseitige Beckenbeschwerden.

Befund: Klein, zart gebaut. Brüste entsprechend dem Alter entwickelt. Unterleib rechts druckempfindlich. Aeussere Genitalien normal. Der trichterige Hymenalsaum rechts hinten lappig eingerissen. Frenulum intact. Bei Entfaltung der grossen Labien zeigt sich im Scheideneingange ein median von der hinteren zur vorderen Wand aufsteigendes, fleischiges Septum, welches die ganze Scheidenlänge in sagittaler Richtung complet durchläuft und sich oben im Scheidengewölbe in leichter Sförmiger Krümmung nach links verzieht. Die schmalen Gewölbe der so gebildeten doppelten Scheide schliessen rechts. und links je eine kurze, virginale, für den Finger wie für das Auge deutlich wahrnehmbare Portio ein mit grübchenförmigem Muttermund. Uterus reclinirt, anteflectirt. Sein wohlabgerundeter Fundus zeigt keine Bicornität. Linkes Ovarium deutlich tastbar. Rechts der äusseren druckempfindlichen Stelle entsprechend eine umschriebene, abgerundete, wallnussgrosse Geschwulst, durch einen dünnen Adnexenstrang mit dem Uterus in Verbindung. Zwei Sonden, von denen je eine in die Vaginalportion der entsprechenden Seite eingeführt wurde, drangen beiderseits $6 \mathrm{Cm}$. weit ein, standen parallel, ohne sich bei Verschiebung gegen einander mit den metallenen Knöpfen zu berühren, wodurch der Nachweis eines completen Uterusseptum geführt wurde. Es wurde auch constatirt, dass Patientin aus beiden Muttermundsöffnungen menstruirte.

Die rechtsseitige Geschwulst verkleinerte sich allmälig bei zweckmässigem Verhalten auf dem Wege der Resorption und liess sich beim Abgangsbefunde der rechte Eierstock in normaler Weise abtasten, so dass auch durch den Verlauf die Annahme einer chronisehentzündlichen Schwellung des rechten Ovariums seine Bestätigung fand.

3. Uterus bicornis duplex mit Vagina duplex.

Marie M...., 18jährige Puella publica aus Prag (klin. Prot. Nr. 596). Seit zwei Jahren normal menstruirt. Nie geboren, nie abortirt. Durch Transferirung von der syphilitischen Abtheilung der Klinik zugewiesen.

Befund: Kräftig gebant, gut genährt. Brustdrüsen gut entwickelt. Warzen, Warzenhöfe schwach pigmentirt. Unterleib nir- 
gends druckempfindlich. Nymphen schlaff. Clitoris etwas vergrössert. Hymen im ganzen rechten hinteren Umfange defect; nur links unten ein langer, scḩmaler Lappen erhalten, welcher sich schräg nach oben rechts einer entsprechenden Stelle des defecten Ringes anfügen lässt, daher möglicherweise in virgine den Mittelbalken eines ursprünglieh doppelt angelegten Hymens gebildet hat. Vom Scheideneingange erstreckt sich, und zwar von der Mitte der vorderen Vaginalwand zum unteren Ende der linken seitlichen Wand ein fleischiges Septum, welches die ganze Scheidenlänge complet durchzieht, und in Folge seiner asymmetrischen Verlaufsrichtung den gesammten Scheidenraum in eine rechte weitere und in eine linke engere Scheide theilt. In beiden weisen Finger und Speculum je eine gut vorspringende Portio mit grübchenförmigem Muttermunde nach. Während die beíden Colla bis in die Höhe des Isthmus gemeinsam verlaufen, divergiren die höher oben gelegenen Abschnitte als spindelförmige Körper, eine tiefe mediane Furche bildend, nach den Seiten hin in der Weise, dass das rechte Horn eine gestreckte Richtung schräg nach rechts, das linke eine sanft abgebogene nach links beibehält. Sondirung: rechtes Horn $5 \mathrm{Cm}$., linkes $71 / 2 \mathrm{Cm}$. lang. Patientin menstruirte ebenfalls, wie die frühere, aus beiden Muttermundsöffnungen.

$\mathrm{Da}$ in dem vorstehenden Falle keine Bildungsstörungen anderer Organe beobachtet wurden, vielmehr die nächsten Nachbarorgane; Urethra, Blase, Mastdarm vollständig normale Beziehungen darboten, so konnte ein Uterus didelphys im Sinne einer wirklichen Verdoppelung des Uterus nicht angenommen werden, zumal eine feste Verbindung der beiden Colla bis in die Höhe des Isthmus bestand. Der hier mitgetheilte Fall lässt sich vielmehr dem von $\mathrm{Cassan}$ beobachteten, in Kussmaul's Werk ${ }^{\mathbf{l}}$ ) und Klebs' Handbuch ${ }^{2}$ ) aufgenommenen Uterus bicornis duplex an die Seite stellen.

4. Uterus bicornis subseptus mit acquirirter Stenose der Scheide.

Rosalie K..., 32jährige Zimmermannsfrau aus Cernosek (klin. Prot. Nr. 4637). Seit dem 13. Lebensjahre viertägig, spärlich, schmerzlos, vierwöchentlich menstruirt. Vor vier Jahren an einer schweren Variola erkrankt; zu gleicher Zeit an einem blutigeitrigen Scheidenausfluss gelitten. Nach Ablauf der Variola tritt Behinderung des Coitus ein wegen Verengerung der Scheide. Dies, $\mathrm{so}_{*}$ wie später auftretende Menstruationsanomalien veranlassten ihren Eintritt in das Spital. In der Reconvalescenz nach den Blattern trat achtmonatliche Menopause ein, verbunden mit zeitweiligen Schmerzen

1) „Von dem Mangel, der Verkümmerung und Verdoppelung der Gebärmutter" u. s. w. S. 170 .

2) Handbuch der pathologischen Anatomie. 4. Lieferung. S. 755. 
im Becken, worauf in Folge einer forcirten Körperbewegung. eine ziemliche Menge flüssigen Blutes auf ein Mal aus der Scheide abgegangen sein soll. Dieser Vorgang soll sich in ganz gleicher Weise noch mehrere Male wiederholt haben, worauf ein normaler Menstruationstypus bis in die letzte Zeit anhielt.

Befund: Kräftig gebaut, mässig genährt, fieberfrei. Unterleib zeigt im Hypogastrium eine leichte Hervorwölbung. Dieser entspricht béi der Palpation eine elastische Resistenz, welche ohne scharfe Umgrenzung drei Querfinger über der Symphyse aufsteigt. Aeussere Genitalien normal u. s. w. Scheide bildet einen nur $3 \mathrm{Cm}$. langen, niedrigen Blindsack, in welchem nach rechts und hinten eine kleine Oeffnung sich befindet, durch die eine Sonde in gerader Richtung nach oben $6 \mathrm{Cm}$. weit eindringt. Uterusverhältnisse nicht zu ermitteln. Bei der Rectumuntersuchung fand man Anus und Mastdarm schlaff und stark ausgedehnt. Es legte dies trotz Leugnen der Patientin die Vermuthung nahe, dass bei der vorhandenen Scheidenstenose Coitusversuche per rectum stattfanden, zumal eine mehrwöchentliche Ruhe im Spitale den Zustand wieder zur Norm zurückführte. Im Douglas'schen Raume tastete man feste, harte Infiltratstränge, welche zu beiden Seiten sich diffus bis an die seitliche Beckenwand fortsetzten.

Anamnese und Befund machten es im hohen Grade wahrscheinlich, dass hier Scheidenstenose und Beckeninfiltrate als Ausgänge einer während der Variolaerkrankung mit diffuser Beckenphlegmone begleiteten Scheidendiphtherie zurückblieben. Nach hinreichender Dilatation der kleinen. Oeffnung des Scheidenblindsackes mit Laminaria wurde nunmehr durch die Untersuchung mit einem Finger constatirt, dass dieselbe die scharfrandige Eingangsöffnung in einen mit contractilen, platten Wandungen ausgekleideten, $21 / 2 \mathrm{Cm}$. langen Kanal bildete, dessen oberes Ende sich durch einen von links her leicht vorspringenden, platten Halbringwulst markirte. Der weiter nach oben vordringende Finger gelangte an einen glattrandigen, fleischigen Sporn, durch welchen zwei Hohlräume geschieden wurden, die mit dem Kanale unmittelbar communicirten. Letztere zeigten, soweit sie sich von innen austasten liessen, allenthalben glatte, mit Schleimhant ausgekleidete Wandungen. Die gleichzeitige äussere Palpation erwies, dass diese muskulösen Wandungen der schon früher erwähnten, elastischen Resistenz über der Symphyse entsprachen. Median unter dem Nabel markirte sich deutlich eine Furche als die Stelle, wo die beiden Uterushörner nach je einer Seite hin divergirten. Letztere hatten eine nach oben abgerundete Form, liessen sich in Anteflexionsstellung von der seitlichen Beckenwand scharf abgrenzen und schienen auch zeitweilig die Wahrnehmung von Contractionserscheinungen zu gestatten. Die genaue Eruirung dieser Verhältnisse war nicht blos durch die Dilatation des Kanales, sondern auch wesentlich dadurch begünstigt, dass in Folge zweckentspreehenden Verhaltens ein Theil der früheren Beckeninfiltrate zur Zeit dieser Untersuchung bereits zur Resorption gelangt war. 
Die Diagnose eines Uterns bicornis subseptus war durch obigen Befund vollkommen klar. Schwieriger gestaltete sich die Deutung des Kanales. Dass derselbe nicht allein der stenosirten Scheidenpartie, sondern auch dem Collum angehören musste, ging schon aus dem Umstande hervor, dass oberhalb des Kanales kein Collum aufzufinden war, indem hier der Sporn Räume schied, welche vermöge ihrer Lagebeziehungen nicht als Colla, sondern als Corpora gedeutet werden mussten. Wieviel aber von dem Kanale der stenosirten Scheide, wieviel dem Collum zukomme, lässt sich aus der Art und Weise der stattgefundenen Zerstörung nicht mehr bestimmen. Der untere Rand des Sporns mochte der Höhe des Isthmus entsprechen. Obgleich dieser platte Rand des Septum, ferner die einfache Bildung des Kanales und des übrigen Scheidenrestes es sehr wahrscheinlich machten, dass ursprünglich ein einfaches Collum da war, so musste andererseits auch dem Umstande Rechnung getragen werden, dass eine Portio vaginalis überhaupt nicht mehr aufzufinden war, auch nicht einmal in Rudimenten, dass dieselbe während der Diphtherie wahrscheinlich mit zerstört wurde und dass sonach ihre ursprüngliche Bildung für die Deutung des ganzen Collum, ob dạsselbe einfach oder doppelt angelegt war, iiberhaupt nicht mehr verwerthet werden konnte.

Die in der Anamnese erwähnten vorübergehenden Retentionszustände mochten wohl darin ihre Erklärung finden, dass die kleine Oeffnung im Scheidenblindsacke sich zeitweise verklebte und erst in Folge von gesteigertem Bauchpressendrucke von Zeit zu Zeit ein spontaner Durchbruch erfolgte. Die Scheidendiphtherie und die mit ihr zusammenhängende Beckenphlegmone hatten in ihren Ausgängen wesentlich dazu beigetragen, die thatsächlichen Verhültnisse zu maskiren, so dass erst nach ausgiebiger Dilatation des stenosirten Kanales und nach partieller Resorption der Beckeninfiltrate eine richtige Erkenntniss aller Details des Bildungsfehlers ermöglicht wurde. Leider musste Patientin gleich nach der Klarstellung der Verhältnisse auf eigenes Andrängen entlassen werden, weshalb zur Zeit weitere Details über' den Verlauf nicht vorliegen.

5. Haematoma septi vaginalis rudimentarii bei einer Schwangeren im fünften Monate.

Josefa N..., 23jührige ledige Magd aus Bohnovie bei Kolin (klin. Prot. Nr. 9254). Seit dem 17. Lebensjahre dreitägig, reichlich, mit Sehmerzen, vierwöehentlich menstruirt. Patientin ist Primigravida im fünften Monate, was sowohl Anamnese wie Befund bestätigen. Veranlassung zu ihrem Eintritte in das Spital gab folgendes Ereigniss :

Vor fünf Tagen trug sie einen mit Kohlen beladenen, schweren Korb bis in den dritten Stock eines Hauses. Dabei bekam sie das schmerzhafte Gefühl, als ob sich etwas aus den äusseren Genitalien herausdränge und fing zugleich heftig aus der Scheide zu bluten an. Eine 
consultirte Hebamme constatirte, dass eine rundliche, ganz schwarz aussehende Geschwulst aus der Scheide hervorgetreten sei. Bei ruhiger Bettlage ging dieselbe wieder zurïck. Die andauernde Blutung veranlasste Patientin, einen Abort befürchtend, ins Gebärhans zu gehen, von wo sie durch die Güte des Herrn Prof. Weber v. Ebenhof der gynäkologischen Klinik zugewiesen wurde.

Localbefund: Aeussere Genitalien normal. Hymen ein nach rechts unten eingerissener Ring. Beim Entfalten der grossen Labien sieht man in dem Scheideneingange einen Theil einer schwarz durchschimmernden Geschwulst. Dieselbe lässt sich mit den Fingern aus der Scheide herausstülpen, wobei man sich überzeugt, dass sie von der Grösse eines Hühnereies auf der schmalen Höhe eines fleischigen Septumrudiments aufsitzt. Dieses letztere, welches der Geschwulst als langgestreckter, schmaler Stiel dient, springt median von den zwei vorderen Dritteln der hinteren Vaginalwand vor, steigt vorn beiläufig zur Höhe einer Fingerbreite klammerartig auf und verflacht sich allmälig nach hinten zu. Die Geschwulst besitzt an ihrer Wölbung sehr zarte, dünne Wandungen, durch welche das geronnene Blut schwarz durchschimmert; dessenungeachtet bot sie eine wohlzusammenhängende, elastisch anzufühlende Masse dar.

Die Diagnose eines Haematoms war in dem vorstehenden Falle sowohl durch die Anamnese als den Befund und weiteren Verlauf gerechtfertigt. Beachtenswerth erscheint hier die Localität des Blutergusses: der obere stumpfe Rand eines rudimentären Vaginals eptum.

Den weiteren Verlauf betreffend, kam es schon am Eintrittstage ins Spital zum spontanen Durchbruch mit Abgang der Coagula, und resorbirte sich der Rest der Geschwulst nach 16tägigem Aufenthalte im Spital bei reinigenden Scheidenverbänden.

Ausser dem Vaginalseptum war keine weitere Bildungsanomalie der Genitalien vorhanden.

Das Haematom katte keine Störung in dem normalen Schwangerschaftsverlaufe veranlasst. 